\title{
Effect of genotype and sex on growth traits, feed utilization and survivability in Nigerian local and Nicholas white exotic turkey
}

*Sola-Ojo, F. E., Ayorinde, K. L., Fayeye, T. R., Ahutu, I. A. and Obadare, A. R. Department of Animal Production, Faculty of Agriculture, University of Ilorin, PMB 1515, Ilorin, Kwara State, Nigeria.

*Corresponding author Email: mofesola1@yahoo.com

\begin{abstract}
Two hundred (200) Nigerian local and Nicholas white (NW) exotic poults (hundred for each breed) were observed from day old to fifteen week for growth traits [Body Weight $(B W)$ and linear body measurements; Body Length (BL), Body Girth (BG), Shank Length (SL) Shank Diameter (SD), Thigh Length (TL), Wing length (WL)], survivability and feed utilization potential (feed intake and feed conversion ratio). There was a significant $(p<0.05)$ genotype effect on $B W$ throughout the experimental period, $B W$ ranged from $120.41 \pm 3.12$ to $2764.00 \pm 32.10 \mathrm{~g}$ and $142.29 \pm 2.94$ to $4601.00 \pm 41.45 \mathrm{~g}$ in Nigerian local and $\mathrm{NW}$ exotic turkey respectively. Sex effect and interaction between genotypes and sex (GxS) on bodyweight were significant $(p<0.05)$ between weeks 7 to 15. Genotypes, sex and GxS effects were also significant $(p<0.05)$ on linear body parameters measured at different ages. Feed intake and feed conversion ratio for the two genotypes were significantly $(p<0.05)$ different. The NW exotic consumed more feed than the local turkey (14.29 to $250.31 \mathrm{~g} v \mathrm{~s} .13 .27$ to $200.47 \mathrm{~g})$ from week 1 to 15 . The Nigerian local poults significantly $(p<0.04)$ had better feed conversion ratio (FCR) from week 1 to 7 (0.17 to 0.21) than the NW exotic (0.20 to 0.23). However, as the poults grow older (from week 8 to 15), the NW exotic had better FCR (0.24 to 0.31) compared to the Nigerian local (0.27 to 0.38).Mortality rate was higher in the $\mathrm{NW}$ exotic (25\%) than the Nigerian local turkey during the study period. This research showed significant genotype, sex and GxS interaction effects on growth traits of turkey. It showed that the NW exotic poults consumed more feed and gained more weight than the Nigerian local from week 1 to 15, had better feed conversion ratio and poorer survivability at older ages (weeks 8 to 15). The Nigerian local consumed smaller quantity of feed and had better FCR at poults stage (weeks 1 to 7). The Nigerian local turkey had better survivability rate throughout the experimental period and can be selected for an improved body weight and better feed utilization at early stage of life.
\end{abstract}

Keywords: Genotype, sex, growth traits, feed utilization, mortality, turkeys

\section{Introduction}

Local or indigenous poultry constitute more than 80 percent of the poultry birds in the country. They are known for adaptation superiority in terms of resistance to disease and other harsh environmental condition (Nwakpu et al., 1999), unfortunately, there has been very little attention from government to improve the contribution of the local poultry to the national GDP. Turkey production is an important and profitable venture in the agricultural industry, with a rising global demand for its products (Case et al., 2010; Ironkwe and Akinola, 2010; Anandah et al., 2012. Yakubu et al., 2013). There are only 1.05 million turkeys in Nigeria and the number is small compared with other poultry species (FAOSTAT, 2010). The local turkey is the fastest growing indigenous poultry and it has the prospect of making more contribution to the Nigerian GDP than any 
other poultry specie.

Recent global restriction in poultry imports accentuated by the threat of avian influenza suggests a renewed interest in indigenous stock. Kallah and Nwagu (1999) suggest the need to develop robust national poultry breeding and development programme. The Food and Agricultural Organization of the UN recognizes rural poultry as an important tool for poverty alleviation in Africa. Economic loss is a common occurrence in many poultry farm today due to in ability of the high producing exotic bred to fully adapt to the harsh tropical environmental conditions. Poultry producers should be aware that losses in production efficiency will occur long before significant mortality rates are observed (Joe and Raymond, 2005). Akinokun (1990) reported that the limitations of exotic breeds are temperature and humidity stress under tropical environment and suggested that a breeding policy in which the introduction of genes of local stock into the exotic stock be initiated. The controlled introduction of new and improved genetic materials into indigenous breeds of birds is expected to speed up genetic progress through the exploitation of hybrid vigour (Adebambo et al., 2006).

There is the need to develop base-line data and modalities of selective breeding of indigenous species without compromising the existence of each unique genetic resource. The needed morphometric and genetic information that could enhance the identification and utilization of superior strains or collections of local turkey for breed development is lacking. Therefore, this study was design to provide information on growth traits and feed utilization potentials of the local and exotic breed of turkey that could serve as a guide in any improvement programme design for the available indigenous local turkey.

\section{Materials and Methods Management of experimental turkey}

One hundred (100) Nigerian local and one hundred (100) Nicholas white exotic poults were obtained at day old from Tolvic farm, Ibadan. On arrival, the poults were weighed, wing tagged according to their genotypes for proper identification, and randomly distributed into eight brooding guards that has been previously labeled according to genotype. Twenty five poults per genotype were placed in each brooding guard throughout the brooding period, there after they were raised in eight partitioned deep litter pens according to their genotype. They were subjected to the same management procedure and fed the same diet throughout the 15 week experimental period. Poults starter mash (0-6 weeks) and turkey growers' mash (7-16 weeks) formulated according to NRC (1994) and containing $28 \%$ and $24 \%$ crude protein respectively were given to the birds. Feed and water were supplied ad libitum, all necessary medications and vaccination were administered accordingly.

\section{Data collection}

Data on growth parameters beginning from first week of age were taken every other week for 15 weeks. Body Weight (BW) was taken in gram using a sensitive weighing scale at early ages and Camry weighing scale at older ages. Body Length (BL), Body Girth (BG), Thigh Length (TL), Shank Length (SL), Keel Length (KL) and Wing Length (WL) were measured in centimeters, with the aid of a measuring tape, while the Shank Diameter (SD) was measured using a pair of Vernier caliper. BL was measured as the length of the body from the base of the comb to the base of the tail around the Uropigial gland, BG was measured as the region of the largest breast expansion while the bird was positioned ventrally, SL was measured using a flexible 
tape rule as the distance from the hock joint to the tarsometarsus digit-3joint, SD was measured using a pair of Vernier caliper at the middle of the left shank of each bird, TL was taken as the distance between the hock joint and the pelvic joint, KL was taken as the length of the cartilaginous keel bone from the cranial to the caudal terminals of the metasternum using a tape rule, WL was measured with the aid of a tape rule with the wing stretched and the measurement taken from the humorous-coracoids junction to tip of the digit.

Survivability was expressed as percentage of the difference between the number of mortality and the number of poults stocked per genotype. Feed intake was obtained as the difference between total feed supplied and the left over at the end of the week. Feed Conversion Ratio (FCR) was calculated as the quantity of feed consumed per unit weight gain.

\section{Statistical Analysis}

Data obtained were subjected to analysis of variance, using the General Linear Model procedure of SPSS (2007). The model used was of the form:

$\mathrm{Y}_{\mathrm{ijk}}=\mu+\mathrm{a}_{\mathrm{i}}+\mathrm{b}_{\mathrm{j}}+(\mathrm{ab})_{\mathrm{ij}}+\mathrm{e}_{\mathrm{ij} k}$.

Where $Y_{\mathrm{ijk}}=$ Measurements taken on the $\mathrm{k}^{\text {th }}$ individual which belongs to the $\mathrm{i}^{\text {th }}$ sex and $\mathrm{j}^{\text {th }}$ genotype.

$\mu=$ overall mean

$a_{i}=$ fixed effect of $i^{\text {th }}$ sex

$b_{j}=$ fixed effect of $j^{\text {th }}$ genotype

$(\mathrm{ab}) \mathrm{ij}=$ Interaction between fixed effects of $i^{\text {th }}$ sex and $j^{\text {th }}$ genotype on poult's body weight

$\mathrm{e}_{\mathrm{ijk}}=$ random residual error.

Significantly different means $(\mathrm{p}<0.05)$ were separated by use of the Duncan's Multiple Range Test(Duncan, 1955).

\section{Results and discussion}

The results showed a significant $(\mathrm{p}<0.05)$ genotype effect on body weight from week 1 to 15 . Body weight increased as the age of the poults increased in Nigerian local and Nicholas white (NW) exotic (Table1). Body weight values in NW exotic were significantly $(p<0.05)$ higher than those of the local turkeys throughout the experimental period and the NW exotic was 18 to $73 \%$ significantly $(\mathrm{p}<0.05)$ higher in body weight than the Nigerian local turkey from week 1 to 15 . Mean Body weight of $120.41 \pm 3.12 \mathrm{~g}$ was obtained for the Nigerian local and $142.29 \pm 2.94 \mathrm{~g}$ for the NW exotic poults at week 1, while $2764.00 \pm 32.104 \mathrm{~g}$ was obtained for the local and $4601.00 \pm 41.45 \mathrm{~g}$ for the NW exotic turkey at week 15. Body weight in male was about $15 \%$ higher in value than that of the female turkey throughout the experimental period. There was a significant difference $(\mathrm{p}<0.05)$ in body weight between sex at weeks 1, 7, 9, 11, 13, 15 with the males having higher values. No significant $(p<0.05)$ differences were observed in body weight of male and female poults at week 3 and 5 . There were significant interactions $(p<0.05)$ effects between sex and genotypes on body weight at different weeks except at week 5, male NW exotic were significantly $(p<0.05)$ heavier than local female turkey from weeks 7 to 15 . However, there was no significant $(p>0.05)$ difference in body weight of NW exotic and local male turkey at week 7 to 15 . 
Effect of genotype and sex on growth traits, feed utilization and survivability in Nigerian local and Nicholas white exotic turkey

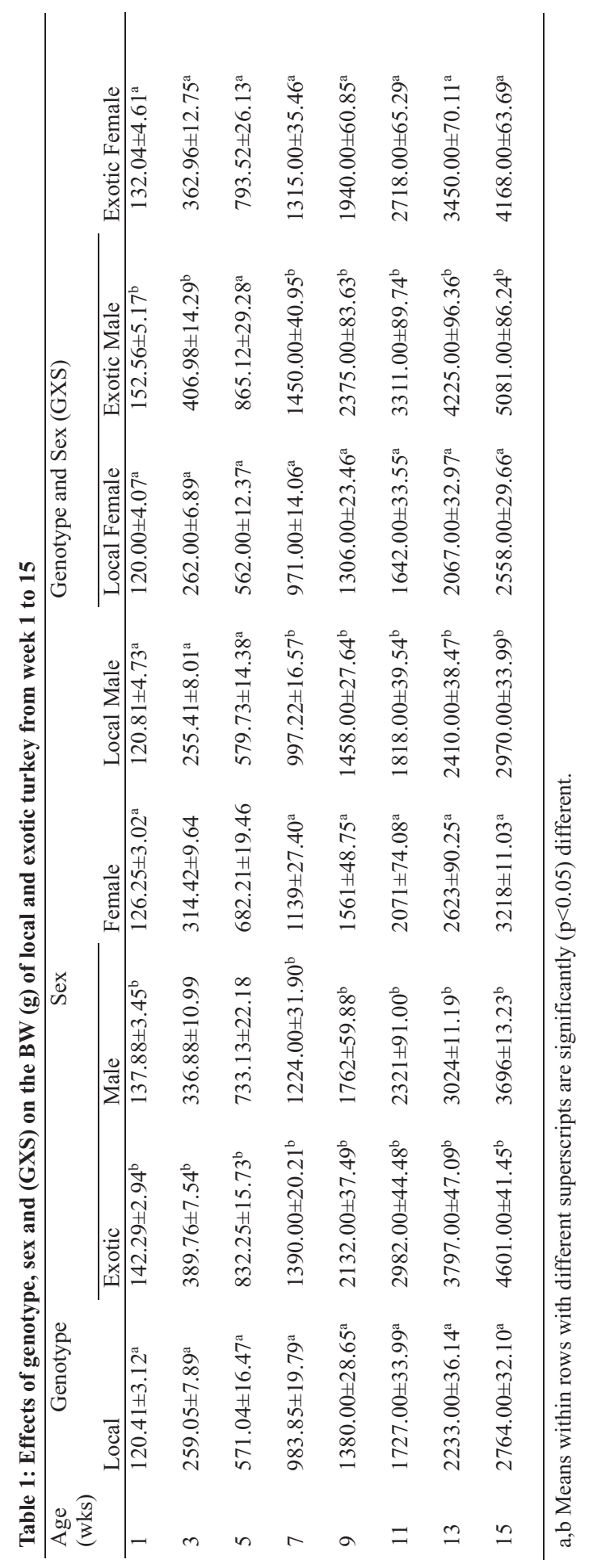


Table 2 shows the effects of genotype, sex and interaction between genotype and sex on body length of turkey studied. Body length measured in different weeks were significantly $(p<0.05)$ affected by the genotype of poults. The NW had higher $(p<0.05)$ body length than the Nigerian local exotic poults. BL ranged from $18.07 \pm 0.12 \mathrm{~cm}$ to $78.62 \pm 0.17 \mathrm{~cm}$ in local and $18.98 \pm 0.12 \mathrm{~cm}$ to $90.09 \pm 0.23 \mathrm{~cm}$ in NW exotic turkey from week 1 to 15 . At week 3 , the local poults had a value of $26.74 \pm 0.21 \mathrm{~cm}$ that was not significantly different from that of NW exotic poults $(30.48 \pm 0.20 \mathrm{~cm})$. The effect of sex on body weight shows that there were significant $(p<0.05)$ differences in body length at week $1,7,9,11,13,15$ with the males having higher body length. No significant $(p>0.05)$ differences in body length of both sexes at week 3 and 5 . There were significant $(p<0.05)$ interactions effects between sex and genotypes on body length at all ages except at 5. No significant $(p>0.05)$ difference in BL between NW exotic and local male at week $5,9,11$, and 15. BL of the NW exotic male was significantly $(p<0.05)$ different from that of local female turkey at all ages except at week 5 .

Table 2: Effects of genotype, sex and (GxS) on the BL (cm) of local and exotic turkey from week 1 to 15

\begin{tabular}{|c|c|c|c|c|c|c|c|c|}
\hline \multirow{2}{*}{$\begin{array}{l}\text { Age } \\
\text { (weeks) }\end{array}$} & \multicolumn{2}{|c|}{ Genotype } & Sex & \multirow[b]{2}{*}{ Female } & \multicolumn{4}{|c|}{ Genotype and Sex (GxS) } \\
\hline & Local & Exotic & Male & & Local Male & $\begin{array}{l}\text { Local } \\
\text { Female }\end{array}$ & Exotic Male & $\begin{array}{l}\text { Exotic } \\
\text { Female }\end{array}$ \\
\hline 1 & $18.07 \pm 0.12^{\mathrm{a}}$ & $18.98 \pm 0.12^{\mathrm{b}}$ & $18.74 \pm 0.13^{\mathrm{b}}$ & $18.32 \pm 0.12^{\mathrm{a}}$ & $18.05 \pm 0.08^{\mathrm{a}}$ & $18.09 \pm 0.07^{\mathrm{a}}$ & $19.34 \pm 0.22^{b}$ & $18.54 \pm 0.19^{\mathrm{a}}$ \\
\hline 3 & $26.74 \pm 0.21$ & $30.48 \pm 0.20$ & $29.06 \pm 1.68$ & $30.20 \pm 1.47$ & $26.87 \pm 0.23^{\mathrm{a}}$ & $26.62 \pm 0.20^{\mathrm{a}}$ & $30.94 \pm 0.37^{\mathrm{b}}$ & $29.79 \pm 0.33^{\mathrm{a}}$ \\
\hline 5 & $39.41 \pm 0.29^{\mathrm{a}}$ & $42.06 \pm 0.28^{b}$ & $41.13 \pm 0.33$ & $40.45 \pm 0.29$ & $39.54 \pm 0.34^{\mathrm{a}}$ & $39.27 \pm 0.29^{\mathrm{a}}$ & $42.49 \pm 0.48^{\mathrm{a}}$ & $41.53 \pm 0.43^{\mathrm{a}}$ \\
\hline 7 & $49.65 \pm 0.24^{\mathrm{a}}$ & $51.14 \pm 0.25^{\mathrm{b}}$ & $50.93 \pm 0.28^{\mathrm{b}}$ & $49.78 \pm 0.24^{\mathrm{a}}$ & $49.92 \pm 0.23^{\mathrm{a}}$ & $49.40 \pm 0.20^{\mathrm{a}}$ & $51.93 \pm 0.48^{\mathrm{b}}$ & $50.17 \pm 0.42^{\mathrm{a}}$ \\
\hline 9 & $57.54 \pm 0.32^{\mathrm{a}}$ & $59.07 \pm 0.41^{\mathrm{b}}$ & $59.09 \pm 0.39^{\mathrm{b}}$ & $57.19 \pm 0.32^{\mathrm{a}}$ & $58.47 \pm 0.41^{\mathrm{b}}$ & $56.68 \pm 0.35^{\mathrm{a}}$ & $60.58 \pm 0.79^{b}$ & $57.87 \pm 0.58^{\mathrm{a}}$ \\
\hline 11 & $65.89 \pm 0.34^{\mathrm{a}}$ & $70.77 \pm 0.44^{\mathrm{b}}$ & $68.95 \pm 0.52^{\mathrm{b}}$ & $66.56 \pm 0.42^{\mathrm{a}}$ & $67.11 \pm 0.58^{\mathrm{b}}$ & $64.75 \pm 0.49^{\mathrm{a}}$ & $72.63 \pm 0.48^{b}$ & $69.29 \pm 0.35^{\mathrm{a}}$ \\
\hline 13 & $76.12 \pm 0.30^{\mathrm{a}}$ & $79.63 \pm 0.39^{b}$ & $79.09 \pm 0.43^{\mathrm{b}}$ & $75.89 \pm 0.35^{\mathrm{a}}$ & $77.69 \pm 0.46^{\mathrm{b}}$ & $74.65 \pm 0.39^{a}$ & $82.01 \pm 0.62^{\mathrm{b}}$ & $77.72 \pm 0.45^{\mathrm{a}}$ \\
\hline 15 & $78.62 \pm 0.17^{\mathrm{a}}$ & $90.09 \pm 0.23^{\mathrm{b}}$ & $84.03 \pm 0.79^{b}$ & $81.95 \pm 0.66^{\mathrm{a}}$ & $80.22 \pm 0.27^{b}$ & $77.03 \pm 0.24^{\mathrm{a}}$ & $91.05 \pm 0.35^{\mathrm{b}}$ & $89.08 \pm 0.26^{\mathrm{a}}$ \\
\hline
\end{tabular}

The effect of genotype on the body girth of local and NW exotic poults was significantly $(p<0.05)$ higher in NW exotic poults. Mean BG ranged from $12.79 \pm 0.07 \mathrm{~cm}$ in Nigerian local poults to $37.50 \pm 0.09 \mathrm{~cm}$ in NW exotic at week 1 , and $13.37 \pm 0.06 \mathrm{~cm}$ in local to $44.63 \pm 0.13 \mathrm{~cm}$ in NW exotic turkey at week 15 (Table 3). The effect of sex on the body girth of poults was not significant $(\mathrm{p}>0.05)$ at weeks 3,5 , and
15, but male poults had higher mean BG. The interaction effect of sex and genotype on the BG were significant $(p<0.05)$ at different ages, at week 1 and 3 , and from week 9 to 15 , male NW exotic had higher $(p<0.05)$ BG mean than local female poults. There was no significant $(p>0.05)$ difference in BG between NW exotic and local male turkey from weeks 9 to 15 . 
Effect of genotype and sex on growth traits, feed utilization and survivability in Nigerian local and Nicholas white exotic turkey

\begin{tabular}{|c|c|c|c|c|c|c|c|c|}
\hline \multirow{2}{*}{$\begin{array}{l}\text { Age in } \\
\text { weeks }\end{array}$} & \multicolumn{2}{|c|}{ Genotype } & \multicolumn{2}{|c|}{ Sex } & \multirow[b]{2}{*}{ Local Male } & \multicolumn{2}{|c|}{ Genotype and Sex (GxS) } & \multirow[b]{2}{*}{$\begin{array}{l}\text { Exotic } \\
\text { Female }\end{array}$} \\
\hline & Local & Exotic & Male & Female & & Local Female & Exotic Male & \\
\hline 1 & $12.79 \pm 0.07^{\mathrm{a}}$ & $13.37 \pm 0.06^{\mathrm{b}}$ & $13.23 \pm 0.08^{b}$ & $12.95 \pm 0.07^{\mathrm{a}}$ & $12.80 \pm 0.08^{\mathrm{a}}$ & $12.78 \pm 0.07^{\mathrm{a}}$ & $13.59 \pm 0.11^{\mathrm{b}}$ & $13.10 \pm 0.09^{\mathrm{a}}$ \\
\hline 3 & $16.39 \pm 0.19^{\mathrm{a}}$ & $20.67 \pm 0.185^{\mathrm{b}}$ & $18.99 \pm 0.31$ & $18.38 \pm .28$ & $16.43 \pm 0.22^{\mathrm{a}}$ & $16.32 \pm 0.19^{\mathrm{a}}$ & $21.19 \pm 0.33^{\mathrm{b}}$ & $20.26 \pm 0.29^{a}$ \\
\hline 5 & $23.01 \pm 0.16^{\mathrm{a}}$ & $26.23 \pm 0.15^{\mathrm{b}}$ & $24.96 \pm 0.24$ & $24.44 \pm 0.21$ & $23.22 \pm 0.20^{\mathrm{a}}$ & $22.79 \pm 0.17^{\mathrm{a}}$ & $26.45 \pm 0.26^{\mathrm{a}}$ & $25.95 \pm 0.23^{\mathrm{a}}$ \\
\hline 7 & $28.47 \pm 0.24^{\mathrm{a}}$ & $29.71 \pm 0.25^{\mathrm{b}}$ & $29.04 \pm 0.18^{\mathrm{b}}$ & $28.34 \pm 015^{\mathrm{a}}$ & $28.52 \pm 0.19^{\mathrm{a}}$ & $28.05 \pm 0.16^{\mathrm{a}}$ & $29.56 \pm 0.29^{\mathrm{a}}$ & $28.64 \pm 0.25^{\mathrm{a}}$ \\
\hline 9 & $29.17 \pm 0.19^{\mathrm{a}}$ & $33.72 \pm 0.25^{\mathrm{b}}$ & $31.53 \pm 0.38^{\mathrm{b}}$ & $30.20 \pm 0.31^{\mathrm{a}}$ & $29.78 \pm 0.23^{\mathrm{b}}$ & $28.59 \pm 0.19^{\mathrm{a}}$ & $35.09 \pm 0.52^{\mathrm{b}}$ & $32.57 \pm 0.38^{\mathrm{a}}$ \\
\hline 11 & $31.70 \pm 0.19^{\mathrm{a}}$ & $37.22 \pm 0.26^{\mathrm{b}}$ & $34.47 \pm 0.43^{b}$ & $33.11 \pm 0.35^{\mathrm{a}}$ & $32.48 \pm 0.32^{\mathrm{b}}$ & $30.99 \pm 0.27^{\mathrm{a}}$ & $38.38 \pm 0.35^{\mathrm{b}}$ & $36.30 \pm 0.26^{\mathrm{a}}$ \\
\hline 13 & $35.87 \pm 0.22^{\mathrm{a}}$ & $41.18 \pm 0.29^{b}$ & $38.82 \pm 0.44^{\mathrm{b}}$ & $36.97 \pm 0.36^{\mathrm{a}}$ & $36.73 \pm 0.27^{\mathrm{b}}$ & $35.06 \pm 0.23^{\mathrm{a}}$ & $43.01 \pm 0.56^{\mathrm{b}}$ & $39.77 \pm 0.41^{\mathrm{a}}$ \\
\hline 15 & $37.50 \pm 0.09^{\mathrm{a}}$ & $44.63 \pm 0.13^{\mathrm{b}}$ & $40.57 \pm 0.49$ & $39.85 \pm 0.41$ & $38.17 \pm 0.15^{\mathrm{b}}$ & $36.84 \pm 0.13^{\mathrm{a}}$ & $44.98 \pm 0.22^{\mathrm{b}}$ & $44.19 \pm 0.16^{\mathrm{a}}$ \\
\hline
\end{tabular}

a,b Means within rows with different superscripts are significantly different $(\mathrm{p}<0.05)$.

Table 4 shows that shank length was significantly $(p<0.05)$ affected by genotype and increased with age of the turkey. Shank length in NW exotic poults was significantly $(p<0.05)$ higher than that of local throughout the experimental period. The effect of sex on shank length shows that there were significant differences $(p<0.05)$ in shank length at week $1,7,9,11,13,15$ with the males having higher values. No significant $(\mathrm{p}<0.05)$ differences in the mean SL at week 3 and 5.

Significant $(\mathrm{p}<0.05)$ interactions existed between genotype and sex on shank length, and male NW exotic had significantly $(p<0.05)$ higher mean value than the local female poults at weeks $1,3,9,11,13$ and 15. There were no significant $(p<0.05)$ differences in the shank length of NW exotic and local male poults at weeks 5 and 7.

Table 4: Effects genotype, sex and (G xS) on the SL (cm) of local and exotic turkey from week 1 to 15

\begin{tabular}{|c|c|c|c|c|c|c|c|c|}
\hline \multirow{2}{*}{$\begin{array}{l}\text { Age } \\
\text { (weeks) }\end{array}$} & \multicolumn{2}{|c|}{ Genotype } & \multicolumn{2}{|l|}{ Sex } & \multirow[b]{2}{*}{ Local Male } & \multicolumn{3}{|c|}{ Genotype and Sex (GxS) } \\
\hline & Local & Exotic & Male & Female & & Local Female & Exotic Male & Exotic Female \\
\hline 1 & $3.14 \pm 0.05^{\mathrm{a}}$ & $3.75 \pm 0.05^{\mathrm{b}}$ & $3.56 \pm 0.06^{\mathrm{b}}$ & $3.35 \pm 0.05^{\mathrm{a}}$ & $3.154 \pm 0.05^{\mathrm{a}}$ & $3.13 \pm 0.04^{\mathrm{a}}$ & $3.90 \pm 0.08^{\mathrm{b}}$ & $3.56 \pm 0.07^{\mathrm{a}}$ \\
\hline 3 & $4.23 \pm 0.08^{\mathrm{a}}$ & $5.04 \pm 0.08^{\mathrm{b}}$ & $4.73 \pm 0.09$ & $4.56 \pm 0.08$ & $4.20 \pm 0.13^{\mathrm{a}}$ & $4.23 \pm 0.11^{\mathrm{a}}$ & $5.18 \pm 0.11^{\mathrm{b}}$ & $4.85 \pm 0.10^{\mathrm{a}}$ \\
\hline 5 & $5.95 \pm 0.08^{\mathrm{a}}$ & $7.09 \pm 0.08^{\mathrm{b}}$ & $6.63 \pm 0.10$ & $6.48 \pm 0.09$ & $5.99 \pm 0.07^{\mathrm{a}}$ & $5.91 \pm 0.06^{\mathrm{a}}$ & $7.18 \pm 0.14^{\mathrm{a}}$ & $7.01 \pm 0.13^{\mathrm{a}}$ \\
\hline 7 & $7.46 \pm 0.06^{\mathrm{a}}$ & $8.13 \pm 0.07^{\mathrm{b}}$ & $7.89 \pm 0.08^{b}$ & $7.66 \pm 0.07^{\mathrm{a}}$ & $7.52 \pm 0.06^{\mathrm{a}}$ & $7.39 \pm 0.05^{\mathrm{a}}$ & $8.26 \pm 0.13^{\mathrm{a}}$ & $7.95 \pm 0.11^{\mathrm{a}}$ \\
\hline 9 & $8.38 \pm 0.06^{\mathrm{a}}$ & $9.44 \pm 0.07^{\mathrm{b}}$ & $8.94 \pm 0.09^{\mathrm{b}}$ & $8.61 \pm 0.08^{\mathrm{a}}$ & $8.51 \pm 0.06^{\mathrm{a}}$ & $8.27 \pm 0.05^{\mathrm{a}}$ & $9.81 \pm 0.16^{\mathrm{b}}$ & $9.13 \pm 0.11^{\mathrm{a}}$ \\
\hline 11 & $9.04 \pm 0.05^{\mathrm{a}}$ & $10.14 \pm 0.07^{\mathrm{b}}$ & $9.61 \pm 0.09^{\mathrm{b}}$ & $9.29 \pm 0.08^{\mathrm{a}}$ & $9.14 \pm 0.08^{\mathrm{a}}$ & $8.93 \pm 0.06^{\mathrm{a}}$ & $10.53 \pm 0.12^{\mathrm{b}}$ & $9.83 \pm 0.08^{\mathrm{a}}$ \\
\hline 13 & $10.07 \pm 0.06^{\mathrm{a}}$ & $12.12 \pm 0.08^{b}$ & $11.11 \pm 0.15^{\mathrm{b}}$ & $10.59 \pm 0.13^{\mathrm{a}}$ & $10.36 \pm 0.09^{b}$ & $9.80 \pm 0.07^{\mathrm{a}}$ & $12.59 \pm 0.14^{\mathrm{b}}$ & $11.75 \pm 0.09^{\mathrm{a}}$ \\
\hline 15 & $10.82 \pm 0.08^{\mathrm{a}}$ & $13.23 \pm 0.10^{\mathrm{b}}$ & $12.12 \pm 0.17^{\mathrm{b}}$ & $11.35 \pm 0.16^{\mathrm{a}}$ & $11.29 \pm 0.09^{\mathrm{b}}$ & $10.34 \pm 0.09^{\mathrm{a}}$ & $13.74 \pm 0.18^{b}$ & $12.78 \pm 0.14^{\mathrm{a}}$ \\
\hline
\end{tabular}

a,b Means within rows with different superscripts are significantly different $(\mathrm{p}<0.05)$.

The NW exotic had significantly $(\mathrm{p}<0.05)$ higher values for shank diameter compared to the local turkey from week 1 to 15 . The value ranged from $0.41 \pm 0.01$ to $1.19 \pm 0.01 \mathrm{~cm}$ in the local and $0.45 \pm 0.01$ to $1.74 \pm 0.01$ in the NW exotic turkey (Table
5). When sex was considered, no significant $(p>0.05)$ differences were observed in the shank diameter at week 3, 5, 7, 11 and 15 . The interaction between genotype and sex were significant except at weeks 5 and 7 . Male NW exotic poults had significantly 
$(p<0.05)$ higher SD than female local at weeks $1,3,9,13$ and 15 . Significant $(p<0.05)$ differences existed in SD of NW exotic and local male poults at week 1,3 and 11.

Table 5: Effects of genotype, sex and (GxS) on the SD (cm) of local and exotic turkey from week 1 to 15

\begin{tabular}{|c|c|c|c|c|c|c|c|c|}
\hline \multirow{2}{*}{$\begin{array}{l}\text { Age in } \\
\text { weeks }\end{array}$} & \multicolumn{2}{|c|}{ Genotype } & \multicolumn{2}{|c|}{ Sex } & \multirow[b]{2}{*}{$\begin{array}{l}\text { Local } \\
\text { Male }\end{array}$} & \multicolumn{2}{|c|}{ Genotype and Sex (GxS) } & \multirow[b]{2}{*}{ Exotic Female } \\
\hline & Local & Exotic & Male & Female & & Local Female & Exotic Male & \\
\hline 1 & $0.41 \pm 0.01^{\mathrm{a}}$ & $0.45 \pm 0.01^{\mathrm{b}}$ & $0.44 \pm 0.01^{\mathrm{b}}$ & $0.43 \pm 0.01^{\mathrm{a}}$ & $0.41 \pm 0.01^{\mathrm{a}}$ & $0.41 \pm 0.02^{\mathrm{a}}$ & $0.47 \pm 0.01^{\mathrm{b}}$ & $0.438 \pm 0.01^{\mathrm{a}}$ \\
\hline 3 & $0.51 \pm 0.01^{\mathrm{a}}$ & $0.62 \pm 0.01^{\mathrm{b}}$ & $0.56 \pm 0.01$ & $0.55 \pm 0.01$ & $0.50 \pm 0.01^{\mathrm{a}}$ & $0.51 \pm 0.01^{\mathrm{a}}$ & $0.64 \pm 0.02^{\mathrm{b}}$ & $0.59 \pm 0.01^{\mathrm{a}}$ \\
\hline 5 & $0.61 \pm 0.01^{\mathrm{a}}$ & $0.78 \pm 0.01^{\mathrm{b}}$ & $0.71 \pm 0.02$ & $0.67 \pm 0.02$ & $0.61 \pm 0.01^{\mathrm{a}}$ & $0.61 \pm 0.01^{\mathrm{a}}$ & $0.80 \pm 0.03^{\mathrm{a}}$ & $0.76 \pm 0.02^{\mathrm{a}}$ \\
\hline 7 & $0.69 \pm 0.01^{\mathrm{a}}$ & $0.89 \pm 0.01^{\mathrm{b}}$ & $0.77 \pm 0.02$ & $0.77 \pm 0.01$ & $0.69 \pm 0.01^{\mathrm{a}}$ & $0.68 \pm 0.01^{\mathrm{a}}$ & $0.89 \pm 0.01^{\mathrm{a}}$ & $0.90 \pm 0.01^{\mathrm{a}}$ \\
\hline 9 & $0.92 \pm 0.01^{\mathrm{a}}$ & $1.06 \pm 0.01^{\mathrm{b}}$ & $1.00 \pm 0.01^{\mathrm{b}}$ & $0.94 \pm 0.01^{\mathrm{a}}$ & $0.95 \pm 0.01^{\mathrm{b}}$ & $0.89 \pm 0.01^{\mathrm{a}}$ & $1.09 \pm 0.02^{\mathrm{b}}$ & $1.03 \pm 0.01^{\mathrm{a}}$ \\
\hline 11 & $1.00 \pm 0.01^{\mathrm{a}}$ & $1.45 \pm 0.01^{\mathrm{b}}$ & $1.19 \pm 0.03$ & $1.16 \pm 0.03$ & $1.03 \pm 0.01^{\mathrm{b}}$ & $0.97 \pm 0.01^{\mathrm{a}}$ & $1.47 \pm 0.02^{\mathrm{a}}$ & $1.43 \pm 0.02^{\mathrm{a}}$ \\
\hline 13 & $1.09 \pm 0.01^{\mathrm{a}}$ & $1.57 \pm 0.01^{\mathrm{b}}$ & $1.32 \pm 0.03^{\mathrm{b}}$ & $1.23 \pm 0.03^{\mathrm{a}}$ & $1.15 \pm 0.01^{\mathrm{b}}$ & $1.041 \pm 0.01^{\mathrm{a}}$ & $1.644 \pm 0.02^{\mathrm{b}}$ & $1.509 \pm 0.02^{\mathrm{a}}$ \\
\hline 15 & $1.19 \pm 0.01^{\mathrm{a}}$ & $1.74 \pm 0.01^{\mathrm{b}}$ & $1.42 \pm 0.04$ & $1.37 \pm 0.03$ & $1.22 \pm 0.02^{\mathrm{b}}$ & $1.16 \pm 0.01^{\mathrm{a}}$ & $1.78 \pm 0.02^{\mathrm{b}}$ & $1.69 \pm 0.01^{\mathrm{a}}$ \\
\hline
\end{tabular}

a,b Means within rows with different superscripts are significantly different $(\mathrm{p}<0.05)$.

Table 6 shows the effect of genotype and sex as well the interaction effects of the two on the keel length. Keel length was significantly $(p<0.05)$ affected by genotypes from week 1 to 15 and ranged from $3.56 \pm 0.04 \mathrm{~cm}$ to $14.15 \pm 0.08 \mathrm{~cm}$ in NW exotic and $3.12 \pm 0.04 \mathrm{~cm}$ to $12.07 \pm 0.06 \mathrm{~cm}$ in the local turkey. The effect of sex on the keel length of poults was not $(p>0.05)$ significant at week $3,5,7$, and 9 , but significant $(p<0.05)$ at weeks $1,11,13$, and 15 , with the male turkeys having higher values. The interaction effect of genotypes and sex on the keel length was significant (p>0.05) at all ages except at weeks 5 and 7. NW exotic male poults had significantly $(p<0.05)$ higher KL than local females at weeks 3, 9, 11 and 13. No significant $(p>0.05)$ difference in the mean KL of NW exotic and local male at week 1,5 and 7. 
Effect of genotype and sex on growth traits, feed utilization and survivability in Nigerian local and Nicholas white exotic turkey

Table 6: Effects of genotype, sex and (GxS) on the KL (cm) of local and exotic turkey from week 1 to 15

\begin{tabular}{|c|c|c|c|c|c|c|c|c|}
\hline \multirow{2}{*}{$\begin{array}{l}\text { Age } \\
\text { (weeks) }\end{array}$} & \multicolumn{2}{|c|}{ Genotype } & \multicolumn{2}{|l|}{ Sex } & \multirow[b]{2}{*}{ Local Male } & \multicolumn{2}{|c|}{ Genotype and Sex (GxS) } & \multirow[b]{2}{*}{$\begin{array}{l}\text { Exotic } \\
\text { Female }\end{array}$} \\
\hline & Local & Exotic & Male & Female & & $\begin{array}{l}\text { Local } \\
\text { Female }\end{array}$ & Exotic Male & \\
\hline 1 & $3.12 \pm 0.04^{\mathrm{a}}$ & $3.56 \pm 0.04^{b}$ & $3.45 \pm 0.05^{b}$ & $3.28 \pm 0.04^{\mathrm{a}}$ & $3.10 \pm 0.03^{\mathrm{a}}$ & $3.14 \pm 0.02^{\mathrm{a}}$ & $3.75 \pm 0.08^{\mathrm{a}}$ & $3.41 \pm 0.07^{b}$ \\
\hline 3 & $4.71 \pm 0.05^{\mathrm{a}}$ & $5.60 \pm 0.05^{\mathrm{b}}$ & $5.26 \pm 0.07$ & $5.09 \pm 0.06$ & $4.74 \pm 0.09^{\mathrm{a}}$ & $4.67 \pm 0.07^{\mathrm{a}}$ & $5.70 \pm 0.07^{b}$ & $5.47 \pm 0.06^{\mathrm{a}}$ \\
\hline 5 & $6.19 \pm 0.08^{\mathrm{a}}$ & $7.36 \pm 0.07^{b}$ & $6.87 \pm 0.10$ & $6.73 \pm 0.09$ & $6.18 \pm 0.08^{\mathrm{a}}$ & $6.19 \pm 0.07^{\mathrm{a}}$ & $7.47 \pm 0.13^{\mathrm{a}}$ & $7.22 \pm 0.12^{\mathrm{a}}$ \\
\hline 7 & $7.81 \pm 0.07^{\mathrm{a}}$ & $8.54 \pm 0.07^{b}$ & $8.26 \pm 0.08$ & $8.06 \pm 0.07$ & $7.86 \pm 0.08^{\mathrm{a}}$ & $7.75 \pm 0.07^{\mathrm{a}}$ & $8.661 \pm 0.12^{\mathrm{a}}$ & $8.379 \pm 0.11^{\mathrm{a}}$ \\
\hline 9 & $8.81 \pm 0.07^{\mathrm{a}}$ & $10.61 \pm 0.09^{\mathrm{b}}$ & $9.67 \pm 0.15$ & $9.31 \pm 0.12$ & $9.05 \pm 0.09^{\mathrm{b}}$ & $8.60 \pm 0.08^{\mathrm{a}}$ & $10.93 \pm 0.19^{b}$ & $10.35 \pm 0.14^{\mathrm{a}}$ \\
\hline 11 & $10.55 \pm 0.08^{\mathrm{a}}$ & $11.88 \pm 0.09^{\mathrm{b}}$ & $11.26 \pm 0.13^{\mathrm{b}}$ & $10.84 \pm 0.10^{\mathrm{a}}$ & $10.78 \pm 0.129^{b}$ & $10.33 \pm 0.11^{\mathrm{a}}$ & $12.200 \pm 0.12^{\mathrm{b}}$ & $11.612 \pm 0.09^{\mathrm{a}}$ \\
\hline 13 & $11.35 \pm 0.06^{\mathrm{a}}$ & $12.86 \pm 0.08^{\mathrm{b}}$ & $12.17 \pm 0.12^{\mathrm{b}}$ & $11.68 \pm 0.10^{\mathrm{a}}$ & $11.64 \pm 0.08^{\mathrm{b}}$ & $11.08 \pm 0.07^{\mathrm{a}}$ & $13.19 \pm 0.13^{b}$ & $12.57 \pm 0.09^{\mathrm{a}}$ \\
\hline 15 & $12.07 \pm 0.06^{\mathrm{a}}$ & $14.15 \pm 0.08^{\mathrm{b}}$ & $13.08 \pm 0.16^{\mathrm{b}}$ & $12.64 \pm 0.13^{\mathrm{a}}$ & $12.42 \pm 0.08^{\mathrm{b}}$ & $11.71 \pm 0.07^{\mathrm{a}}$ & $14.32 \pm 0.14^{\mathrm{a}}$ & $13.98 \pm 0.10^{\mathrm{a}}$ \\
\hline
\end{tabular}

Genotype, sex and interaction between genotype and sex effects on thigh length are as shown in Table 7. The thigh length increased with age of the poults, the values of TL in NW exotic were significantly $(p<0.05)$ higher than those of the local poults from week 1 to 15 . The effect of sex on the TL of the turkey shows that there was no significant difference $(p>0.05)$ in the thigh length of both the male and female at weeks 3, 7, 9 and 15. However, TL in male was significantly $(\mathrm{p}<0.05)$ higher at weeks $1,5,11$ and 13 . The value obtained ranged from $7.83 \pm 0.08 \mathrm{~cm}$ at week 1 to $24.43 \pm 0.37 \mathrm{~cm}$ at week 15 compared to that of the females that ranged from $7.59 \pm 0.07 \mathrm{~cm}$ at week 1 to $24.05 \pm 0.31 \mathrm{~cm}$ at week 15.The interaction effect of genotype and sex was significant $(p<0.05)$ at different ages. NW exotic male had significantly $(p<0.05)$ higher TL mean than local female poults at weeks 1, 3, 7, 9 and 13. At week 5 there was no significant $(p>0.05)$ difference in the TL of NW exotic and local male poults.

\begin{tabular}{|c|c|c|c|c|c|c|c|c|}
\hline \multirow{2}{*}{$\begin{array}{l}\text { Age } \\
\text { (weeks) }\end{array}$} & \multicolumn{2}{|c|}{ Genotype } & \multicolumn{2}{|l|}{ Sex } & \multirow[b]{2}{*}{ Local Male } & \multicolumn{3}{|c|}{ Genotype and Sex (GxS) } \\
\hline & Local & Exotic & Male & Female & & $\begin{array}{l}\text { Local } \\
\text { Female }\end{array}$ & Exotic Male & $\begin{array}{l}\text { Exotic } \\
\text { Female }\end{array}$ \\
\hline 1 & $7.16 \pm 0.06^{\mathrm{a}}$ & $8.22 \pm 0.05^{b}$ & $7.83 \pm 0.08^{b}$ & $7.59 \pm 0.07^{\mathrm{a}}$ & $7.20 \pm 0.09^{\mathrm{a}}$ & $7.12 \pm 0.08^{\mathrm{a}}$ & $8.38 \pm 0.08^{b}$ & $8.03 \pm 0.07^{\mathrm{a}}$ \\
\hline 3 & $9.82 \pm 0.11^{\mathrm{a}}$ & $11.48 \pm 0.10^{\mathrm{b}}$ & $10.81 \pm 0.14$ & $10.55 \pm 0.13^{\mathrm{a}}$ & $9.82 \pm 0.16^{\mathrm{a}}$ & $9.82 \pm 0.13^{\mathrm{a}}$ & $11.67 \pm 0.17^{\mathrm{b}}$ & $11.20 \pm 0.15^{\mathrm{a}}$ \\
\hline 5 & $13.23 \pm 0.14^{\mathrm{a}}$ & $14.85 \pm 0.13^{\mathrm{b}}$ & $14.30 \pm 0.17^{\mathrm{b}}$ & $13.84 \pm 0.15^{\mathrm{a}}$ & $13.36 \pm 0.17^{\mathrm{a}}$ & $13.09 \pm 0.15^{\mathrm{a}}$ & $15.12 \pm 0.23^{\mathrm{a}}$ & $14.53 \pm 0.20$ \\
\hline 7 & $16.09 \pm 0.08^{\mathrm{a}}$ & $18.25 \pm 0.08^{\mathrm{b}}$ & $17.31 \pm 0.16$ & $16.97 \pm 0.13$ & $16.14 \pm 0.05^{\mathrm{a}}$ & $16.04 \pm 0.04^{\mathrm{a}}$ & $18.49 \pm 0.18^{\mathrm{b}}$ & $17.94 \pm 0.16^{\mathrm{a}}$ \\
\hline 9 & $18.06 \pm 0.12^{\mathrm{a}}$ & $20.69 \pm 0.16^{\mathrm{b}}$ & $19.24 \pm 0.23$ & $18.84 \pm 0.19$ & $18.22 \pm 0.16^{\mathrm{a}}$ & $17.89 \pm 0.13^{\mathrm{a}}$ & $21.27 \pm 0.32^{\mathrm{b}}$ & $20.25 \pm 0.24^{\mathrm{a}}$ \\
\hline 11 & $20.85 \pm 0.14^{\mathrm{a}}$ & $22.83 \pm 0.18^{\mathrm{b}}$ & $22.08 \pm 0.21^{\mathrm{b}}$ & $21.16 \pm 0.17^{\mathrm{a}}$ & $21.21 \pm 0.21^{\mathrm{b}}$ & $20.49 \pm 0.18^{\mathrm{a}}$ & $23.84 \pm 0.25^{\mathrm{a}}$ & $22.15 \pm 0.18^{b}$ \\
\hline 13 & $21.39 \pm 0.14^{\mathrm{a}}$ & $25.41 \pm 0.18^{\mathrm{b}}$ & $23.41 \pm 0.30^{\mathrm{b}}$ & $22.40 \pm 0.25^{\mathrm{a}}$ & $21.98 \pm 0.19^{\mathrm{b}}$ & $20.86 \pm 0.16^{\mathrm{a}}$ & $26.23 \pm 0.29^{\mathrm{b}}$ & $24.67 \pm 0.21^{\mathrm{a}}$ \\
\hline 15 & $22.19 \pm 0.09^{\mathrm{a}}$ & $27.57 \pm 0.12^{\mathrm{b}}$ & $24.43 \pm 0.37$ & $24.05 \pm 0.31$ & $22.59 \pm 0.09^{\mathrm{b}}$ & $21.82 \pm 0.08^{\mathrm{a}}$ & $27.91 \pm 0.25^{\mathrm{a}}$ & $27.24 \pm 0.19^{\mathrm{a}}$ \\
\hline
\end{tabular}

a,b Means within rows with different superscripts are significantly different $(\mathrm{p}<0.05)$. 
Table 8 shows the effect of sex and genotype on the wing length of local and NW exotic poults, WL was significantly $(p<0.05)$ affected by genotype with a value that ranged from $10.01 \pm 0.07 \mathrm{~cm}$ to $33.87 \pm 0.15 \mathrm{~cm}$ in NW exotic and $8.54 \pm 0.07 \mathrm{~cm}$ to $29.75 \pm 0.11 \mathrm{~cm}$ in the local turkey from week 1 to 15 . The effect of sex on the wing length showed no significant
( $p>0.05$ ) differences except at week 13. The interaction effect of sex and genotype on the WL of turkey was significant $(p<0.05)$ at weeks $9,11,13$ and 15 . The wing length of the NW exotic male turkey was significantly $(\mathrm{p}<0.05)$ higher than local female at weeks 11 and 13 . From week 1 to 15 , there was no significant $(p>0.05)$ difference in the WL of NW exotic and local male turkey.

Table 8: Effects of genotype, sex and (GxS) on the WL (cm) of local and exotic turkey from week 1 to 15

\begin{tabular}{|c|c|c|c|c|c|c|c|c|}
\hline \multirow{2}{*}{$\begin{array}{l}\text { Age } \\
\text { in } \\
\text { weeks }\end{array}$} & \multicolumn{2}{|c|}{ Genotype } & \multicolumn{2}{|l|}{ Sex } & \multirow[b]{2}{*}{ Local Male } & \multicolumn{2}{|c|}{ Genotype and Sex (GxS) } & \multirow{2}{*}{ Exotic Female } \\
\hline & Local & Exotic & Male & Female & & Local Female & Exotic Male & \\
\hline 1 & $8.54 \pm 0.07^{\mathrm{a}}$ & $10.01 \pm 0.07^{\mathrm{b}}$ & $9.42 \pm 0.11$ & $9.17 \pm 0.95$ & $8.54 \pm 0.06^{\mathrm{a}}$ & $8.53 \pm 0.05^{\mathrm{a}}$ & $10.17 \pm 0.13^{\mathrm{a}}$ & $9.76 \pm 0.12^{\mathrm{a}}$ \\
\hline 3 & $11.69 \pm 0.11^{\mathrm{a}}$ & $13.73 \pm 0.11^{\mathrm{b}}$ & $12.84 \pm 0.16$ & $12.68 \pm 0.14$ & $11.65 \pm 0.19^{\mathrm{a}}$ & $11.74 \pm 0.17^{\mathrm{a}}$ & $13.86 \pm 0.15^{\mathrm{a}}$ & $13.51 \pm 0.13^{\mathrm{a}}$ \\
\hline 5 & $17.23 \pm 0.17^{\mathrm{a}}$ & $19.83 \pm 0.16^{\mathrm{b}}$ & $18.77 \pm 0.22$ & $18.42 \pm 0.20$ & $17.281 \pm 0.22^{\mathrm{a}}$ & $17.168 \pm 0.16^{\mathrm{a}}$ & $20.06 \pm 0.26^{\mathrm{a}}$ & $19.58 \pm 0.23^{\mathrm{a}}$ \\
\hline 7 & $17.90 \pm 0.09^{\mathrm{a}}$ & $20.82 \pm 0.09^{b}$ & $19.48 \pm 0.19$ & $19.19 \pm 0.17$ & $18.03 \pm 0.11^{\mathrm{a}}$ & $17.78 \pm 0.09^{\mathrm{a}}$ & $20.93 \pm 0.17^{\mathrm{a}}$ & $20.65 \pm 0.14^{\mathrm{a}}$ \\
\hline 9 & $20.38 \pm 0.12^{\mathrm{a}}$ & $25.95 \pm 0.15^{\mathrm{b}}$ & $22.52 \pm 0.39$ & $22.40 \pm 0.32$ & $20.61 \pm 0.14^{\mathrm{a}}$ & $20.17 \pm 0.12^{\mathrm{b}}$ & $26.18 \pm 0.31^{\mathrm{a}}$ & $25.75 \pm 0.22^{\mathrm{a}}$ \\
\hline 11 & $23.01 \pm 0.21^{\mathrm{a}}$ & $29.23 \pm 0.28^{\mathrm{b}}$ & $25.93 \pm 0.48$ & $24.79 \pm 0.39$ & $23.642 \pm 0.34^{\mathrm{b}}$ & $22.48 \pm 0.29^{\mathrm{a}}$ & $30.41 \pm 0.37^{b}$ & $28.291 \pm 0.27^{\mathrm{a}}$ \\
\hline 13 & $28.66 \pm 0.10^{\mathrm{a}}$ & $31.62 \pm 0.13^{\mathrm{b}}$ & $30.17 \pm 0.23^{b}$ & $29.36 \pm 0.19^{\mathrm{a}}$ & $29.15 \pm 0.16^{\mathrm{b}}$ & $28.18 \pm 0.14^{\mathrm{a}}$ & $32.16 \pm 0.17^{b}$ & $31.11 \pm 0.13^{\mathrm{a}}$ \\
\hline 15 & $29.75 \pm 0.11^{\mathrm{a}}$ & $33.87 \pm 0.15^{\mathrm{b}}$ & $31.57 \pm 0.31$ & $31.05 \pm 0.26$ & $30.21 \pm 0.13^{\mathrm{a}}$ & $29.29 \pm 0.12^{\mathrm{b}}$ & $34.09 \pm 0.30^{\mathrm{a}}$ & $33.59 \pm 0.22^{\mathrm{a}}$ \\
\hline
\end{tabular}

a,b Means within rows with different superscripts on the same row are significantly $(\mathrm{p}<0.05)$ different

The significant $(\mathrm{p}<0.05)$ difference reported in body weight of poults from week 1 to 15 is in contrary to the report of Ilori et al. (2010) where the author stated that the local turkey breed had a non-significantly different body weight at week 1 among the breeds of turkey studied. The findings that exotic turkey showed higher and significant body weight when compared with local turkey, and also that the body weight of both genotypes of turkey increased with age corroborate the findings of Ilori et al. (2016). The difference and superiority exhibited by the exotic turkey suggested that it had a better growth potential than the local turkey. This might be due to the fact that the breed had gone through intense selection for higher growth rate as stated by Ilori et al. (2010). Lower growth traits value reported for the Nigerian local turkey is expected since our indigenous poultry have gone through more of natural selection for survivability in the tropical climate rather than artificial selection for better performance and productivity Ibe (1998).

The results obtained from the linear body parameters measured in this study justify the report of Gous, (1997) that growth is normally accompanied by an orderly sequence of maturational changes and involves accretion of protein and increase in length and size, not just an increase in body weight. In this study, the mean body weight and the linear body measurements increased as the age of the turkey increased. The results of this finding also revealed that the NW exotic male and local male turkeys generally had higher and significantly 
Effect of genotype and sex on growth traits, feed utilization and survivability in Nigerian local and Nicholas white exotic turkey

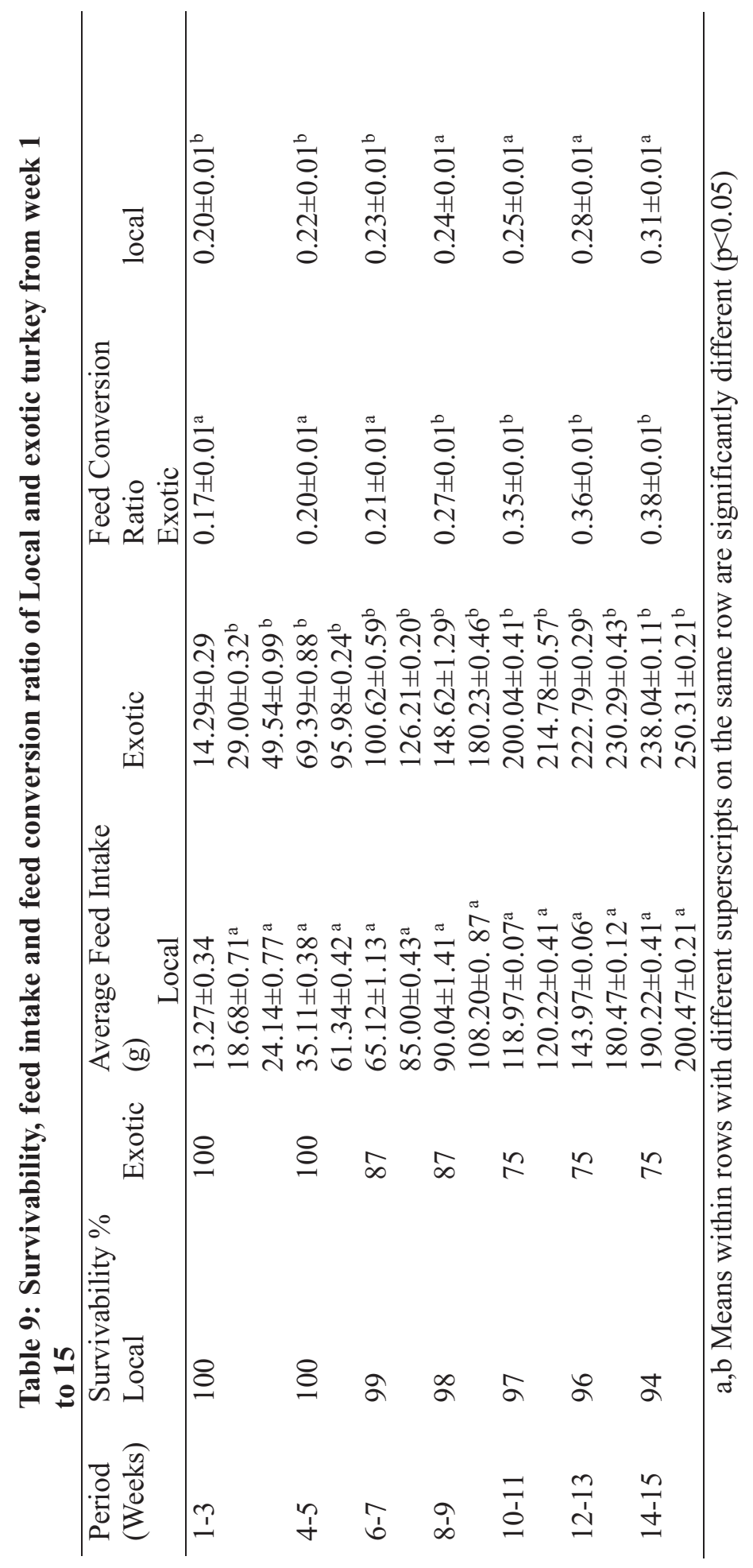


different values in growth parameters measured at all ages when compared to their female counterparts, in cases where the females had higher values, the difference were not significant. This supports the report of Garcia et al. (1991) and Ikeobi et al. (1995) that sexual dimorphism was in favour of males in the performance of strains of birds. Fayeye et al. (2006) attributed this difference to genetic effect of sex which arises from the male physiological activities. It was also been reported by Ibe and Nwosu (1999) that sex differences were usually due to different hormonal profile, aggressiveness and dominance traits exhibited by males especially when both sexes are reared together.

Table 9 shows the survivability of the two genotypes of turkey studied. No mortality was recorded for the two genotypes from week 1 to 5 . Survivability of the local turkey was higher than that of the NW exotic turkey from week 6 to 15 . The local turkey had a mortality range of 1 to $6 \%$, while the NW exotic turkey had a mortality range of $13-25 \%$ from 6 to 15 weeks of age. The mortality recorded indicates that the Nigerian local turkey had better survivability than the Nicholas white exotic turkey as the experimental birds grow older. The genotype effect on feed intake and feed conversion ratio is also shown in Table 9. Feed intake was significantly $(\mathrm{p}<0.05)$ affected by genotype throughout the experimental period except at week 1 . Feed intake increased as the age of the turkey increased, the NW exotic consumed significantly $(\mathrm{p}<0.05)$ higher than the local turkeys except at week 1 where there was no significant $(p>0.05)$ difference in the quantity of feed consumed. The average feed intake per day per bird was $14.29 \pm 0.29 \mathrm{~g}$ in NW exotic turkey and $13.27 \pm 0.34 \mathrm{~g}$ in the local turkey at week 1
$250.31 \pm 0.21 \mathrm{~g}$ in NW exotic turkey and $200.47 \pm 0.21 \mathrm{~g}$ in the local turkey at week 15.

The feed conversion ratio in both genotype of turkey studied indicated that the local turkey had a feed conversion ratio that ranged from $0.17 \pm 0.01$ at week 1 to $0.38 \pm 0.01$ at week 15 , while the exotic had a feed conversion ratio ranging from $0.20 \pm 0.00$ at week 1 to $0.31 \pm 0.00$ at week 15. The effect of genotype on feed conversion ratio was significant $(\mathrm{p}<0.05)$ throughout the experimental period. The local turkeys had lower but better FCR from week 1 to 7 and higher but poorer feed conversion ratio from week 8 to 15 , while the NW exotic turkey had higher but poorer feed conversion ratio from week 1 to 7 and lower but better feed conversion ratio from week 8 to 15 . These results showed that the Nigerian local turkey consumed less feed and gained less weight than the Nicholas White turkey from week 1 to 15 , but had better feed conversion ratio when they were young and poorer FCR at older ages.

\section{Conclusion}

This study was able to make comparisons between Nigerian local and NW exotic turkey and provide useful and extensive baseline data that can be used in any selection and breeding program design for improvement of growth traits in the indigenous turkey. It showed that the NW exotic had significantly higher growth traits than the local turkey which indicates superiority of growth parameters by the NW exotic turkey. From the study, significant effects of genotype, sex and interaction between genotype and sex on body weight and all the linear body parts measured occurred in the NW exotic and local turkey at different ages. The NW exotic turkey consumed higher quantity of feed at all ages, had better feed conversion ratio from 
Effect of genotype and sex on growth traits, feed utilization and survivability in Nigerian local and Nicholas white exotic turkey

week 8 to 15 , while the Nigerian local turkey consumed significantly lower quantity of feed throughout the experimental period, but had better feed conversion ratio from week 1 to 6 , thus can be selected for an improved growth traits at early stage of life.

\section{Acknowledgement}

The authors would like to thank the management of the University of Ilorin for the provision of Nigeria Universities Doctoral Thesis Award Scheme (NUDTAS, 2010) supervisor award grant used for this research.

\section{References}

Adebambo, A. O., Ozoje, M. O., Adebambo, O. A. and Abiola, S. S. 2006. Genetic variations in growth performance of Giriraja, Indian white leghorn and improved indigenous chicken Genotypes in South West Nigeria. Nigerian Journal of Genetics, 20: 9-21.

Akinokun, O. 1990. An evaluation of exotic and indigenous chickens as genetic materials for development of rural poultry in Africa. In: Proceedings of an International Workshop on rural poultry development in Africa held at the Obafemi Awolowo University, IleIfe, Nigeria: 36-61.

Anandahl, M. A., Jagatheesan, P. N. R., Kumar, P. S., Rajarajan, G. and Paramasivam, A. 2012. Effect of egg weight on egg traits and hatching performance of turkey (Meleagris gallopavo) eggs. Iranian Journal of Applied Animal Science 2: 391-395.

Case, L. A., Miller, S. P. and Wood, B. J. 2010. Factors affecting breast meat yield in turkeys. World's Poultry
Science Journal 66: 189-202.

Duncan, D. B. 1955. Multiple Range and Multiple F-tests. Biometrics 11, pp. 1-42.

FAOSTAT, 2010. Food and Agriculture Organization of the United Nations. (Available at http://faostat.fao.org/default.aspx) .Accessed 19 July, 2011.

Fayeye, T. R., Ayorinde, K. L., Ojo, V. and Adeshina, O. M. 2006. Frequency and influence of some major genes on body weight and body size parameters of Nigerian $1 \mathrm{o} \mathrm{c} \mathrm{a} 1 \quad \mathrm{c} \mathrm{h}$ i c k e $\mathrm{n} \mathrm{s}$. LRRD183.2006http//www.cipav.or g.co.lrrd.18/3/faye18037.htm.

Garcia, E. A., Mendes, A. A., Curi, P. K., Silva, A. B., Da, P. and Gonzalez. 1991. Effect of line and diet on growth and carcass yield of broiler. Animal Breeding Abstract., 61, (8) 1993. Geflugekd: 21-28.

Gous, R. 1997. Understanding growth and carcass development. In: World's Poultry Science Elsevier publication, 13: 466-48.

Ibe, S. N. and Nwosu, U. F. 1999. Influence of naked neck and frizzle genes on early growth of chickens. Book of Proceedings. 26th Annual NSAP Conference 21-25 March, Ilorin Nigeria: 292-295.

Ikeobi, C. O. N., Peters, S. O. and Ebozoje, M. O. 1995. Sexual dimorphism in two strains of broiler chicken. Nigerian Journal of Genetics, 10: 16-22.

Ilori, B. M., Peters, S. O. Ikeobi, C. O. N., Bamgbose, A. M. Isidahomen, C. E. and Ozoje, M. O. 2010. Comparative Assessment of Growth in Pure and Crossbred Turkeys in a Humid Tropical Environment International 
Journal of Poultry Science, 9 (4): 368-375.

Ilori, B. M., Akano, K., Durosaro, S. O., Adebambo, A. O. and Ozoje, $M$. O. (2016). Estimates of repeatability for growth traits of pure and crossbred turkeys in the tropics. Nigerian journal of Animal production, 43 (1): 27-36.

Ironkwe, M. O. and Akinola, L. F. 2010. Profitability of turkey productuion in Ahoada East local government area of Rivers State, Nigeria. Continental Journal of Agricultural Science 4: 38-41.

Joe, G. B. and Raymond, L. H. 2005. Hot weather management in the poultry house. Division of Agricultural Sciences and Natural Resources. Oklahoma Cooperative Extension Service-ANSI- 8205. http://www.osuextra.com and.
Kallah, M. S. and Nwagu, B. I. 1999. Nigerian indigenous chicken. What future!. Genetics and food security in Nigeria. A 25 year commemorative publication of the Genetics Society of Nigeria (19741999): 194-199.

Nwakpu, P. E., Odo, B. L., Omeje, S. S., Akpa, M, and Edoga, C. C. 1999. Hatching performance of three strains of layer type of chicken and their lines, Proceedings of $26^{\text {th }} \quad N S A P$ conference :21-23.

SPSS 2007. Statistical package for the social sciences. SPSS Inc., 444 Michigan Avenue, Chicago, IL60611, USA.

Yakubu, A., Abimiku, K. Musa-Azara, I. S., Idahor, K. O. and Akinsola, $O$. M. 2013. Assessment of flock structure, preference in selection and traits of economic importance of domestic turkey (Meleagris gallopavo) genetic resources in Nasarawa State, Nigeria. Livestock Research for Rural Development. www.lrrd.cipav.org

Received: $20^{\text {th }}$ September, 2016 Accepted: $28^{\text {th }}$ January, 2017 